Research Paper

\title{
New Clinical Features and Dosimetric Predictor Identification for Symptomatic Radiation Pneumonitis after Tangential Irradiation in Breast Cancer Patients
}

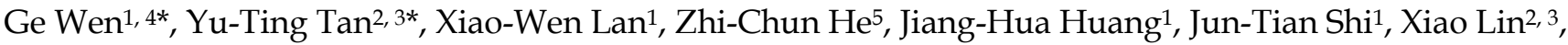 \\ Xiao-Bo Huang1, 2,3凶 \\ 1. Department of Radiation Oncology, Sun Yat-sen Memorial Hospital, Sun Yat-sen University, Guangzhou 510120, Guangdong, P.R. China; \\ 2. Breast Tumor Center, Sun Yat-sen Memorial Hospital, Sun Yat-sen University, Guangzhou 510120, Guangdong, P.R. China; \\ 3. Guangdong Provincial Key Laboratory of Malignant Tumor Epigenetics and Gene Regulation, Medical Research Center, Sun Yat-sen Memorial Hospital, \\ Sun Yat-sen University, Guangzhou 510120, Guangdong, P.R. China; \\ 4. Department of Radiation Oncology, the Third Affiliated Hospital of Guangzhou Medical University, Guangzhou 510150, Guangdong, P.R. China; \\ 5. Department of Radiation Oncology, Sun Yat-sen University Cancer Center, State Key Laboratory of Oncology in South China, Collaborative Innovation \\ Center of Cancer Medicine, Guangzhou 510060, Guangdong, P.R. China. \\ * These authors contributed equally to this work. \\ $\triangle$ Corresponding author: Xiao-Bo Huang, M.D., Ph.D., Department of Radiation Oncology, Sun Yat-sen Memorial Hospital, Sun Yat-sen University, No. 107, \\ Yanjiang West Road, Yuexiu District, Guangzhou 510120, Guangdong, P.R. China; Telephone: +0086-020-34071346; E-mail: huangxb903km@163.com \\ (c) Ivyspring International Publisher. This is an open access article distributed under the terms of the Creative Commons Attribution (CC BY-NC) license \\ (https://creativecommons.org/licenses/by-nc/4.0/). See http://ivyspring.com/terms for full terms and conditions.
}

Received: 2017.05.24; Accepted: 2017.08.08; Published: 2017.10.17

\begin{abstract}
Background: Tangential irradiation is the most popular postoperative radiotherapy technique for breast cancer. However, irradiation has been related to symptomatic radiation pneumonitis (SRP), which decreases the quality of life of patients. This study investigated the clinical features and dosimetric parameters related to SRP of the ipsilateral lung to identify risk factors for SRP in breast cancer patients after three-dimensional conformal radiation therapy (3D-CRT) with tangential fields.

Material and Methods: A total of 515 breast cancer patients were evaluated and divided into two groups: the local-regional irradiation group (259 patients) and the simple local irradiation group (256 patients). Clinical symptoms were registered and patient data collected. The relationship between the incidence of SRP and dosimetric parameters for the ipsilateral lung was assessed within 6 months after 3D-CRT. Dosimetric parameters were compared using $t$ tests. The dosimetric predictors for SRP were estimated using a logistic regression model and receiver operating characteristic curve analysis.

Results: In total, 19 patients (3.7\%) developed grade 2 SRP. In the local-regional irradiation group, the probability of SRP in the lung body was greater than that in the lung apex (3.9\% vs. $1.5 \%)$. V20 and $\mathrm{V} 30$ were independent predictors for SRP in the local-regional irradiation group (odds ratio $=$ 1.152 and 1.439 , both $p=0.030$ ), whereas only V20 was an independent predictor of SRP in the simple local irradiation group (odds ratio $=1.351, p=0.001$ ). With $39.8 \%$ as the optimal threshold for $\mathrm{V} 20$ and $25.7 \%$ for $\mathrm{V} 30$ for local-regional irradiation, SRP could be predicted with an accuracy of $80.3 \%$ and $79.9 \%$, a sensitivity of $61.5 \%$ and $69.2 \%$, and a specificity of $81.3 \%$ and $80.5 \%$, respectively. With $20.2 \%$ as the optimal V20 threshold for simple local irradiation, SRP could be predicted with an accuracy of $88.7 \%$, a sensitivity of $83.3 \%$ and a specificity of $89.6 \%$.

Conclusions: SRP has become a rare complication with mild symptoms and occurs mainly in the lung body. V20 and V30 may be useful dosimetric predictors to evaluate SRP risk of the ipsilateral lung in breast cancer.
\end{abstract}

Key words: Breast cancer; Radiation pneumonitis; Radiotherapy; Tangential irradiation. 


\section{Introduction}

Breast cancer (BC) is one of the most common tumours in women, and its morbidity has increased in recent years, especially in China. Postoperative radiotherapy (RT) is widely used in the treatment of $\mathrm{BC}$ not only to reduce the risk of loco-regional recurrence but also to improve overall survival [1,2]. The main indication for $\mathrm{RT}$ in $\mathrm{BC}$ is high risk for recurrence, such as stage T1-2 patients undergoing breast-conserving surgery (BCS) or stage $\geq \mathrm{T} 3$ patients undergoing modified radical mastectomy (MRM) or with positive axillary lymph nodes. At present, the main irradiation technique uses tangential fields for the whole breast/chest wall and the ipsilateral supraclavicular field for patients with positive lymph node involvement.

However, lung tissue is inevitably affected by irradiation during $\mathrm{RT}$, and radiation-induced lung injury, one of the most common complications of postoperative RT for BC, seriously affects patient quality of life and even increases non-cancer mortality $[3,4]$. Radiation-induced lung injury typically presents in three sequential pathologic phases: an exudative phase, an organizing or proliferative phase, and a chronic fibrotic phase [5]. In the clinic, these injuries are categorized as two distinct, subsequent clinical phases: acute pneumonitis and chronic fibrosis. Symptomatic radiation pneumonitis (SRP) results from acute exudation in the alveolar space and the migration of inflammatory cells. It usually occurs within 3-6 months after RT, and the symptoms include cough, sputum, dyspnoea, fever, malaise, and hypoxia. SRP can regress completely with significant supportive measures, including steroids, oxygen supplementation, or even mechanical ventilation. Even with treatment, SRP can evolve into fibrosis when present at a severe grade and remains potentially fatal. Pulmonary fibrosis is a late injury due to interstitial damage involving the parenchyma and pleura. If the threshold for potential complete restitution is crossed, SRP will develop into pulmonary fibrosis within 6-12 months. Therefore, prevention of SRP is better than treatment. It is important but difficult to accurately predict SRP risk before RT for BC. It has been reported that the risk of SRP is proportional to the volume of lung irradiated as well as the irradiation dose $[5,6]$.

The development of three-dimensional conformal radiotherapy (3D-CRT) has improved radiation delivery by increasing the accuracy of tumour targeting and enhancing the dosimetric evaluation of normal tissues. Along these lines, many models have been investigated to predict SRP for thoracic tumours [7-9].
Previous studies have primarily focused on the factors correlated with SRP, such as patient clinical features, dosimetric parameters of the bilateral lung and combined therapies [10, 11]. However, BC patients mostly receive tangential irradiation of the whole breast/chest wall, and only the ipsilateral lung is irradiated. Thus, precise dose-volume histogram $(\mathrm{DVH})$ evidence and data for the prediction of SRP in the unilateral lung in $\mathrm{BC}$ are still lacking. The present study had two main aims: 1) to explore the clinical features of SRP and 2) to evaluate dosimetric parameters with the purpose of identifying useful predictors of SRP in BC patients receiving 3D-CRT with tangential fields.

\section{Material and Methods}

\section{Patient selection and clinical features}

This retrospective analysis was conducted on 515 consecutive BC patients treated with BCS or MRM and postoperative RT between March, 2012, and September, 2015.

The inclusion criteria were as follows: (1) age $\leq$ 70 years old; (2) postoperative pathological diagnosis of breast carcinoma; (3) postoperative RT initiated after completion of chemotherapy for patients receiving adjuvant chemotherapy; (4) no previous history of respiratory illness and no abnormalities on chest X-ray and/or chest computed tomography (CT) examination prior to RT; (5) treatment with 3D-CRT or "field-in-field" forward intensity modulated radiation therapy (IMRT) and tangential photon fields with conventional fractions (1.8-2.0 Gy/fraction) designed for irradiation of the whole breast/chest wall; (6) RT was designed and accomplished by a full-time radiation oncologist; (7) dosimetric parameters of DVH were available; (8) a minimum of 6 months of follow-up data were available, and there was survival without any recurrence or new metastasis within 6 months after RT.

Data on clinical features and dosimetric parameters of DVH were collected. In total, 230 patients had a right breast tumour: 82 underwent BCS, and 148 underwent MRM. Additionally, 285 patients had a left breast tumour: 92 underwent BCS, and 193 underwent MRM. The median age was 44 years (range, 20-69 years), 294 patients were staged pTis-2N0-1M0, and 221 patients were staged pT1-4N2-3M1. Overall, 20 patients were stage 0, 60 were stage I, 220 were stage II, 213 were stage III, and 2 were stage IV.

\section{Radiotherapy treatment}

RT was scheduled to start within 1 month after chemotherapy and within 8 weeks after surgery for patients without chemotherapy. Planning CT images 
were obtained using a spiral CT scanner (Brilliance Big Bore, Philips) with the scan range extended from the cricothyroid to the diaphragm, covering the entire thoracic and supraclavicular fossa region at $5-\mathrm{mm}$ intervals. The patient was placed in the treatment position during image acquisition, and the images were imported into the ADAC Pinnacle3 8.0 (Philips, Eindhoven, Netherlands) treatment planning system. Target volumes were outlined according to the criteria of the International Commission of Radiation Units and Measurements Reports 50 and $62[12,13]$.

The treatment consisted of two opposed wedged tangential photon beams ( 4 or $6 \mathrm{MV}$ ) for the whole breast/chest wall. The total prescribed dose was 45-50 Gy delivered in 1.8-2.0 Gy/fraction at five fractions/week. For patients who underwent MRM, an 1.0-cm-depth tissue equivalent bolus was used on the chest wall. For patients who underwent BCS, an additional boost irradiation (10 Gy in five fractions) was delivered to the tumour bed using an electron field (6 or $9 \mathrm{MeV}$ ). The supraclavicular field was treated with an anterior oblique photon field to a total dose of 45-50 Gy. The border between the tangential fields and the supraclavicular field was typically at the level of $5 \mathrm{~mm}$ under the inferior aspect of the clavicular head. In 15 patients, the internal mammary lymph nodes (IMNs) were covered with either extended tangential photon fields or a separate oblique electron field to a total dose of 45-50 Gy.

\section{Other adjuvant treatment}

Adjuvant chemotherapy was performed before irradiation and included anthracycline-based regimens (CEF/CAF, EC/AC) or taxane-based regimens (TEC/TAC, TE/TA or EC/AC-T). Selective oestrogen receptor modulators (SERMs) or aromatase inhibitors (AIs) were used as endocrine therapy. Some patients with human epidermal growth factor receptor-2 (HER-2)-positive cancer were administered trastuzumab.

\section{Evaluation of SRP}

To determine SRP levels, chest CT images obtained at baseline (before RT) were compared with those obtained during RT or within 6 months after treatment. Follow-up examinations included chest CT and physical examinations. SRP was diagnosed in patients who developed dry cough, productive cough (sputum), dyspnoea, fever or hypoxia with characteristic CT manifestations in infiltrating range (presence of reticular markings, ground glass opacities, inter/intra lobular septal linear thickening and lung consolidation) corresponding to the radiation portal instead of the pulmonary segments and lobes, with the exception of pulmonary infection, tuberculosis and pulmonary metastasis [14, 15]. SRP was graded according to the Common Terminology Criteria for Adverse Events v4.03 [16].

In order to reflect the relationship between SRP area and RT site, the lung was divided into two regions (i.e., the apex and body) according to the border of the tangential fields and the supraclavicular field. The field above the border was defined as the lung apex, and the field below the border was defined as the lung body $[9,17]$.

\section{Dosimetric parameter selection}

The patients were divided into two groups as follows: 1 . the local-regional irradiation group included 259 patients treated with tangential fields at the breast/chest wall ( \pm IMN irradiation) and irradiation of the supraclavicular field, 2. the simple local irradiation group included 256 patients treated with only tangential fields at the breast/chest wall. Dosimetric parameters were calculated based on the cumulative $\mathrm{DVH}$, including the percentage of lung volume that received doses exceeding $5,10,20,30$, and 40 Gy (V5, V10, V20, V30, and V40, respectively) as well as the mean dose $\left(D_{\text {mean }}\right)$ and the max dose $\left(D_{\max }\right)$ to the ipsilateral lung.

\section{Statistical analysis}

The SPSS statistical software package (version 22.0; IBM Corporation, Armonk, NY, USA) was used to establish a database. Dosimetric parameters of patients with and without SRP were compared using $t$ tests. All statistical tests were two-sided, and $p<0.05$ was considered statistically significant. The dosimetric predictors for SRP were estimated using a logistic regression model (Method = Backward: LR; probability of entry: 0.05) and receiver operating characteristic (ROC) curve analysis.

\section{Results}

\section{Follow-up outcomes and manifestations of SRP}

The last follow-up was performed in July 2016. The median follow-up time was 34 months (range, 8-50 months) after the completion of RT.

Radiologic changes due to radiation (grade 1) were detected in $76.1 \%(197 / 259)$ of patients in the local-regional irradiation group and 69.5\% (178/256) of patients in the simple local irradiation group. Nineteen patients $(3.7 \%, 19 / 515)$ developed SRP (grade 2). The affected sites were only the lung apex in 3 patients, only the lung body in 9 patients and both the lung apex and body in 1 patient (the total involved probability of SRP in the lung apex was $1.5 \%$ and 3.9\% in the lung body) in the local-regional irradiation group and the lung body in 6 patients $(2.3 \%)$ in the 
simple local irradiation group. All cases of SRP occurred from 1 to 4 months after the completion of RT. The symptoms were cough in 9 patients $(47.4 \%)$, sputum in 7 patients $(36.8 \%)$, and fever in 3 patients $(15.8 \%)$. No patients presented with dyspnoea or hypoxia.

\section{Dosimetric parameters for SRP risk}

The mean values of various dosimetric parameters were calculated. V5, V10, V20, V30, V40, and $\mathrm{D}_{\text {mean }}$ showed significant correlations with SRP by univariate analysis (all $p<0.05$ ) in both groups (Table 1). In multivariate analysis, V20 and V30 were independent predictors of SRP in the local-regional irradiation group, whereas V20 was the only independent predictor of SRP in the simple local irradiation group (Table 2).

\section{ROC curve analysis of the local-regional irradiation group}

As shown in Figure 1A, the area under the ROC curve was 0.766 for V20 $(p=0.001)$, and the optimal V20 threshold to predict SRP was $39.8 \%$. To verify the accuracy of this threshold, the 13 patients with SRP were divided into two subgroups based on V20 value: 5 patients were in the $\mathrm{V} 20<39.8 \%$ group, and 8 patients were in the V20 $\geq 39.8 \%$ group. The incidence of SRP was $2.4 \%(5 / 205)$ in the former group and $14.8 \%(8 / 54)$ in the latter group $(p=0.001)$. Using the threshold, SRP could be predicted with an accuracy of $80.3 \%$, a sensitivity of $61.5 \%$ and a specificity of $81.3 \%$.

The ROC area was 0.782 for V30 $(p=0.001)$, and the optimal threshold for V30 was $25.7 \%$. Accordingly, the 13 patients with SRP were divided into two subgroups based on V30 value: 4 patients were in the $\mathrm{V} 30<25.7 \%$ group, and 9 patients were in the $\mathrm{V} 30 \geq 25.7 \%$ group. The incidence of SRP was $2.0 \%$ $(4 / 202)$ in the former group and $15.8 \%(9 / 57)$ in the latter group $(p<0.001)$. Using this threshold, SRP could be predicted with an accuracy of $79.9 \%$, a sensitivity of $69.2 \%$ and a specificity of $80.5 \%$.

\section{ROC curve analysis of the simple local irradiation group}

As shown in Figure 1B, the ROC area was 0.885 for V20 $(p=0.001)$, and the optimal threshold of V20 was $20.2 \%$. Accordingly, the 6 patients with SRP were divided into two subgroups based on V20 value: 2 patients were in the $<20.2 \%$ group, and 4 patients were in the $\mathrm{V} 20 \geq 20.2 \%$ group. The incidence of SRP was $0.9 \%(2 / 225)$ in the former group and $12.9 \%$ $(4 / 31)$ in the latter group $(p<0.001)$. Using this threshold, SRP could be predicted with an accuracy of $88.7 \%$, a sensitivity of $83.3 \%$ and a specificity of $89.6 \%$.

\section{Discussion}

SRP significantly affects the quality of life of BC patients who receive postoperative RT and could even lead to the development of pulmonary fibrosis and lung function decline. Previous studies have shown that SRP can manifest as cough, sputum, or fever, and in severe cases, patients can present with dyspnoea and hypoxia [10, 14]. In the cohort examined in the present study, the manifestations of SRP were milder than expected and may have been atypical. Most patients with SRP developed only respiratory symptoms consisting of cough $(47.4 \%)$ and sputum (36.8\%). The incidence of fever (15.8\%) was low, and no patients exhibited symptoms of grade 3 or higher SRP, such as dyspnoea or hypoxia.

Table 2. Multivariate analysis of dosimetric parameters potentially associated with symptomatic radiation pneumonitis (SRP).

\begin{tabular}{lccc}
\hline Factors & Odds ratio & $95 \%$ CI & $p$ value \\
\hline Local-regional irradiation & & & \\
V20 $(\%)$ & 1.152 & $1.014-1.308$ & 0.030 \\
V30 $(\%)$ & 1.439 & $1.036-2.000$ & 0.030 \\
Simple local irradiation & & & \\
V20 $(\%)$ & 1.351 & $1.139-1.603$ & 0.001 \\
\hline
\end{tabular}

Table 1. Univariate analysis of dosimetric parameters potentially associated with symptomatic radiation pneumonitis (SRP).

\begin{tabular}{|c|c|c|c|c|c|c|}
\hline \multirow[b]{2}{*}{ Factors } & \multicolumn{3}{|c|}{ Local-regional irradiation } & \multicolumn{3}{|c|}{ Simple local irradiation } \\
\hline & $\begin{array}{l}\text { SRP } \\
(\text { mean } \pm S D)\end{array}$ & $\begin{array}{l}\text { No-SRP } \\
(\text { mean } \pm S D)\end{array}$ & $p$ value & $\begin{array}{l}\text { SRP } \\
(\text { mean } \pm S D)\end{array}$ & $\begin{array}{l}\text { No-SRP } \\
(\text { mean } \pm S D)\end{array}$ & $p$ value \\
\hline V5 (\%) & $58.86 \pm 8.28$ & $46.35 \pm 13.88$ & $<0.001$ & $38.05 \pm 12.35$ & $25.89 \pm 6.13$ & $<0.001$ \\
\hline V10 (\%) & $48.15 \pm 9.32$ & $36.64 \pm 12.17$ & 0.001 & $29.54 \pm 8.76$ & $19.64 \pm 5.06$ & $<0.001$ \\
\hline V20 (\%) & $41.97 \pm 11.77$ & $29.59 \pm 11.44$ & $<0.001$ & $21.88 \pm 4.83$ & $14.77 \pm 3.96$ & $<0.001$ \\
\hline V30 (\%) & $27.27 \pm 5.30$ & $20.23 \pm 6.86$ & $<0.001$ & $17.71 \pm 4.51$ & $11.78 \pm 3.74$ & $<0.001$ \\
\hline V40 (\%) & $17.92 \pm 7.08$ & $11.81 \pm 5.90$ & $<0.001$ & $12.09 \pm 4.92$ & $8.13 \pm 3.25$ & 0.004 \\
\hline $\mathrm{D}_{\text {mean }}(\mathrm{cGy})$ & $1911.84 \pm 422.81$ & $1423.04 \pm 460.89$ & $<0.001$ & $1397.38 \pm 310.39$ & $979.19 \pm 372.71$ & 0.007 \\
\hline $\mathrm{D}_{\max }(\mathrm{cGy})$ & $5870.11 \pm 537.17$ & $5740.25 \pm 369.88$ & 0.230 & $5762.67 \pm 407.69$ & $5462.21 \pm 473.40$ & 0.125 \\
\hline Ipsilateral lung volume (cc) & $1351.24 \pm 320.77$ & $1219.58 \pm 285.42$ & 0.108 & $1270.94 \pm 200.08$ & $1180.44 \pm 273.42$ & 0.665 \\
\hline Bilateral lung volume (cc) & $2602.17 \pm 762.56$ & $2373.93 \pm 479.50$ & 0.306 & $2326.05 \pm 576.79$ & $2328.45 \pm 458.54$ & 0.792 \\
\hline
\end{tabular}



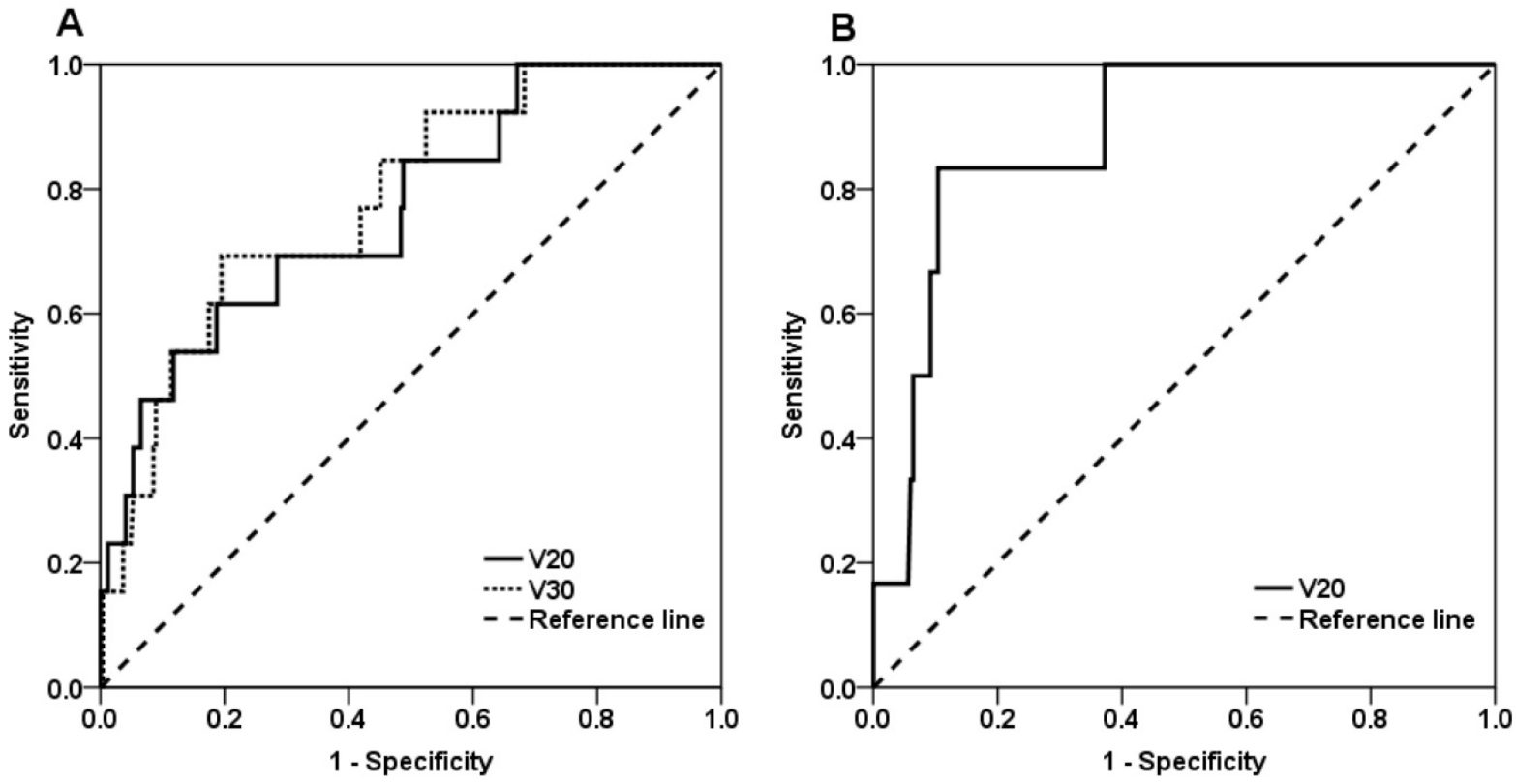

Figure 1. ROC curves for the predictors of symptomatic radiation pneumonitis. (A) V20 and V30 in the local-regional irradiation group and (B) V20 in the simple local irradiation group.

Previous reports have shown that irradiation of the breast/chest wall with supraclavicular field leads to an increased incidence of SRP $[18,19]$. A retrospective study of 613 patients found that the incidence of SRP was higher in patients receiving loco-regional RT compared to those treated only with local RT (4.1\% vs. $0.9 \%$, respectively) [18]. A meta-analysis also found that supraclavicular field irradiation significantly affects the incidence of SRP (odds ratio $=5.07 ; 95 \% \mathrm{CI}=1.95-13.22$ ) [19]. Similar to previous reports, our results showed that the incidence of SRP was $3.7 \%$ for all patients and that local-regional irradiation was associated with a higher incidence of SRP $(5.0 \%, 13 / 259)$ compared with simple local irradiation (2.3\%). In previous studies, the radiologic-induced inflammation was more likely to appear in the lung apex than in other regions following loco-regional RT [17]. However, in the local-regional irradiation group in the present study, SRP mainly occurred in the lung body: the incidence of SRP was $3.9 \%$ and $1.5 \%$ in the lung body and lung apex, respectively. This difference could be due to the improvement of technology for breast cancer RT. In the past, postoperative RT was generally applied to the breast/chest wall as well as to the lymph nodes in the axilla, supraclavicular. It led to a higher degree of apical lung exposure [2]. Furthermore, in the past, conventional RT (for which 3D dose distributions are not available) resulted in high-dose regions at the interface between the tangential and the supraclavicular fields as well as a higher percentage of lung apex volume receiving high doses. These limitations have been effectively resolved with the emergence of 3D-CRT, through which the dose-volume distribution can be directly evaluated and adjusted. In addition, SRP incidence is also correlated with chemotherapy and IMN irradiation. A retrospective study of 1,624 patients receiving RT after BCS showed that the incidence of SRP was higher in patients who received chemotherapy compared to those without chemotherapy $(3 \%$ vs. $0.5 \%$, respectively, $p=0.0001)$. When patients received local-regional irradiation with concurrent chemotherapy, the incidence of SRP was $8.8 \%$ compared with $1.3 \%$ for patients receiving sequential chemoradiotherapy $(p=0.002)$ [20]. Since concurrent chemoradiotherapy is no longer advocated for the combined therapy of $\mathrm{BC}$, the patients in this study were treated with sequential chemoradiotherapy. Thus, the role of IMN irradiation in patients with positive axillary lymph nodes remains controversial. Choi et al. [21] reported that SRP occurred in $6.5 \%$ of patients who underwent IMN irradiation and in 3.3\% of patients without IMN irradiation $(p=0.047)$ and considered that IMN irradiation resulted in increased radiation exposure to the lung and a higher risk of developing SRP. Therefore, it is important to carefully select patients for treatment with IMN irradiation and adopt more accurate radiotherapy technology in clinical practice. In our study, the proportion of patients receiving IMN irradiation was only 5.8\% $(15 / 259)$.

It is important to reduce the incidence of SRP in $\mathrm{BC}$ by taking preventive measures based on the above-discussed clinical features. There is a critical need to explore the effective value of DVH parameters 
in predicting SRP and guiding the design and evaluation of treatment planning. In a 1991 report published by the Photon Treatment Planning Collaborative Working Group, the bilateral lung was treated as a single organ, and data on tolerance doses were gathered primarily from patients with intrathoracic tumours, such as lung cancer, oesophageal cancer, or Hodgkin's disease [22]. Therefore, previous studies examining SRP have also treated the bilateral lung as a single critical organ in the evaluation of treatment planning, and V20 was used as an indicator to predict SRP risk [23-26]. A study of 99 patients with inoperable non-small cell lung cancer found that V20 was an independent predictor of SRP in multivariate analysis; the incidence of SRP was $0 \%, 7 \%, 13 \%$ and $36 \%$ in patients with V20 values $<22 \%, 22-31 \%, 32-40 \%$, and $>40 \%$, respectively. Severe ( $\geq$ grade 3 ) pneumonitis emerged when V20 > 32\% [25]. Asakura et al. [26] analysed 37 oesophageal cancer patients treated with definitive concurrent chemoradiotherapy and found that the optimal V20 threshold to predict SRP was 30.5\% according to ROC curve analysis. The incidence of grade $\geq 2$ SRP was $64.3 \%$ in patients with V20 $\geq 30.5 \%$ and $17.4 \%$ in patients with V20 $<30.5 \%$. Some studies have shown that V30 is also highly correlated with SRP [27-29]. A retrospective analysis of 201 lung cancer patients who underwent 3D-CRT showed that V30 was independently associated with SRP $(p=$ 0.003 ); the incidence of SRP was $6 \%$ and $24 \%$ in patients with V30 values $\leq 18 \%$ and $>18 \%$, respectively [27]. In patients with intrathoracic tumours, the irradiated region tends to be more central and involve the bilateral lungs, whereas in BC, the region irradiated with tangential fields is generally thin and peripherally located in the ipsilateral lung. Therefore, the lung DVH criteria used to evaluate SRP risk in RT treatment planning for intrathoracic tumours may not be directly applicable to BC. Kutcher et al. [30] reported that it appears adequate to consider paired organs as either one unit or as entirely independent units. Therefore, this study treated the ipsilateral lung as an independent organ to explore the dose-volume predictors of SRP for BC. According to previous studies, there is a correlation between increasing V20 value and SRP [9, 31, 32]. Lind et al. [9] analysed the relationship between dosimetric factors and SRP in the ipsilateral lung with ROC curve analysis in 128 patients with early-stage BC after postoperative RT. They found that V20, age, and reduced pre-RT functional level were independently related to SRP, and the area under the ROC curve of V20 was 0.69 in all patents and increased to 0.76 in patients $<55$ years old. In the present study, both the V20 and the V30 of the ipsilateral lung were independently associated with SRP in the local-regional irradiation group, and the ROC areas and optimal thresholds for SRP in relation to V20 and V30 were 0.766 and 0.782 and $39.8 \%$ and $25.7 \%$, respectively. Furthermore, in the simple local irradiation group, V20 was the only independent predictor associated with SRP. The ROC area for V20 was 0.885 , and the optimal threshold was $20.2 \%$. The present findings suggest that V20 may be an effective evaluation indicator to predict SRP for early-stage BC receiving only tangential irradiation of the breast/chest wall. Moreover, V30 will obviously increase when patients need to receive additional supraclavicular $( \pm \mathrm{IMN})$ field irradiation, as this will give rise to a larger lung exposure volume. Thus, it is necessary to consider V30 when designing and evaluating treatment planning in this situation. Therefore, for patients receiving local-regional irradiation, we recommend the use of ipsilateral lung $\mathrm{V} 20<39.8 \%$ and V30 $<25.7 \%$ for the overall evaluation of radiotherapy planning and the use of ipsilateral lung V20 $<20.2 \%$ for further evaluation of the breast/chest wall tangential fields alone. In addition, some recent studies of intrathoracic tumours also found that the risk of SRP significantly correlated with low dose-volume metrics (such as V5, V10, and V13) [33, 34]. Schallenkamp et al. [35] considered that larger lung volumes treated with lower doses may be more critical in predicting SRP than smaller volumes treated with higher doses, and suggested that the radiation of intrathoracic tumours should be planned with caution, especially when using IMRT delivering low radiation doses to large lung volumes. By contrast, in BC, the region irradiated with tangential fields is thin and peripherally located in the ipsilateral lung. Moreover, the lung apex is irradiated in the supraclavicular field, and the general belief is that structural changes of a similar volume in the lung apex have less consequence for whole-lung function than those in the centre or base of the lung because of regional physiologic differences in perfusion and ventilation [17]. In agreement with this, the present study did not identify V5 or V10 as independent predictors of SRP.

We recognize that this study has certain limitations. It is a retrospective analysis, and therefore there may be an inherent selection bias due to the limited number of cases available at a single institution. Larger prospective clinical studies are expected to provide a higher level of evidence.

\section{Conclusions}

In summary, with the popularity of 3D-CRT, SRP has become a rare complication with mild symptoms and occurs mainly in the lung body. Our 
findings suggest that a V20 threshold of $39.8 \%$ and a V30 threshold of $25.7 \%$ for BC patients receiving local-regional irradiation and a V20 threshold of $20.2 \%$ for patients receiving simple local irradiation of the ipsilateral lung are the optimal cut-offs for predicting SRP. However, caution should be taken when using these thresholds, as they were derived from a limited number of patients and need further confirmation in a larger sample of BC patients receiving ipsilateral RT. In addition, with new technologies such as IMRT and hypofractionated RT being increasingly applied for $\mathrm{BC}$, additional studies of corresponding dose-volume parameters should be performed to better guide clinical practice.

\section{Abbreviations}

BC: Breast cancer; RT: Radiotherapy; BCS: Breast-conserving surgery; MRM: Modified radical mastectomy; SRP: Symptomatic radiation pneumonitis; 3D-CRT: Three-dimensional conformal radiation therapy; DVH: Dose-volume histogram; CT: Computed tomography; IMRT: Intensity modulated radiation therapy; IMNs: Internal mammary lymph nodes; CEF: Cyclophosphamide, epirubicin, and fluorouracil; CAF: Cyclophosphamide, doxorubicin, and fluorouracil; EC: Epirubicin and cyclophosphamide; AC: Doxorubicin and cyclophosphamide; TEC: Docetaxel, epirubicin, and cyclophosphamide; TAC: Docetaxel, doxorubicin, and cyclophosphamide; TE: Docetaxel and epirubicin; TA: Docetaxel and doxorubicin; T: Docetaxel; SERMs: Selective oestrogen receptor modulators; AIs: Aromatase inhibitors; HER-2: Human epidermal growth factor receptor-2; $\mathrm{D}_{\text {mean: }}$ : Mean dose; $\mathrm{D}_{\max }$ : Max dose; ROC: Receiver operating characteristic.

\section{Competing Interests}

The authors have declared that no competing interest exists.

\section{References}

1. EBCTCG (Early Breast Cancer Trialists' Collaborative Group), McGale P, Taylor C, Correa C, Cutter D, Duane F, et al. Effect of radiotherapy after mastectomy and axillary surgery on 10-year recurrence and 20-year breast cancer mortality: meta-analysis of individual patient data for 8135 women in 22 randomised trials. Lancet. 2014; 383: 2127-35.

2. Clarke M, Collins R, Darby S, Davies C, Elphinstone P, Evans V, et al. Effects of radiotherapy and of differences in the extent of surgery for early breast cancer on local recurrence and 15-year survival: an overview of the randomised trials. Lancet. 2005; 366: 2087-106.

3. Darby SC, Ewertz M, McGale P, Bennet AM, Blom-Goldman U, Bronnum D, et al. Risk of ischemic heart disease in women after radiotherapy for breast cancer. N Engl J Med. 2013; 368: 987-98.

4. Little MP. A review of non-cancer effects, especially circulatory and ocular diseases. Radiat Environ Biophys. 2013; 52: 435-49.

5. Davis SD, Yankelevitz DF, Henschke CI. Radiation effects on the lung: clinical features, pathology, and imaging findings. AJR Am J Roentgenol. 1992; 159: 1157-64.

6. Lind P. Clinical relevance of pulmonary toxicity in adjuvant breast cancer irradiation. Acta Oncol. 2006; 45: 13-5.
7. Oh YT, Noh OK, Jang H, Chun M, Park KJ, Park KJ, et al. The features of radiation induced lung fibrosis related with dosimetric parameters. Radiother Oncol. 2012; 102: 343-6.

8. Zhao L, Ji W, Ou G, Lv J, Liang J, Feng Q, et al. Risk factors for radiation-induced lung toxicity in patients with non-small cell lung cancer who received postoperative radiation therapy. Lung Cancer. 2012; 77: 326-30.

9. Lind PA, Wennberg B, Gagliardi G, Rosfors S, Blom-Goldman U, Lidestahl A, et al. ROC curves and evaluation of radiation-induced pulmonary toxicity in breast cancer. Int J Radiat Oncol Biol Phys. 2006; 64: 765-70.

10. Ogo E, Komaki R, Abe T, Uchida M, Fujimoto K, Suzuki G, et al. The clinical characteristics and non-steroidal treatment for radiation-induced bronchiolitis obliterans organizing pneumonia syndrome after breast-conserving therapy. Radiother Oncol. 2010; 97: 95-100.

11. Kubo A, Osaki K, Kawanaka T, Furutani S, Ikushima H, Nishitani H. Risk factors for radiation pneumonitis caused by whole breast irradiation following breast-conserving surgery. J Med Invest. 2009; 56: 99-110.

12. ICRU report. Vol. 50: Prescribing, recording, and reporting photon beam therapy. Maryland: International Commission on Radiation Units and Measurements; 1993

13. ICRU Report. Vol. 62: Prescribing, recording, and reporting photon beam therapy (supplement to ICRU report 50). Maryland: International Commission on Radiation Units and Measurements; 1999.

14. Kano A, Ujita M, Kobayashi M, Sunakawa Y, Shirahama J, Harada T, et al. Radiographic and CT features of radiation-induced organizing pneumonia syndrome after breast-conserving therapy. Jpn J Radiol. 2012; 30: 128-36.

15. Krengli M, Sacco M, Loi G, Masini L, Ferrante D, Gambaro G, et al. Pulmonary changes after radiotherapy for conservative treatment of breast cancer: a prospective study. Int J Radiat Oncol Biol Phys. 2008; 70: 1460-7.

16. U.S. Department of Health and Human Services. Common Terminology $\begin{array}{lllll}\text { Criteria for } & \text { Adverse }\end{array}$ http://evs.nci.nih.gov/ftp1/CTCAE/CTCAE_4.03_201 0-06-14_QuickReference_8.5x11.pdf. Accessed 22 Jul 2016.

17. Wennberg B, Gagliardi G, Sundbom L, Svane G, Lind P. Early response of lung in breast cancer irradiation: radiologic density changes measured by CT and symptomatic radiation pneumonitis. Int J Radiat Oncol Biol Phys. 2002; 52: 1196-206

18. Lind PA, Marks LB, Hardenbergh PH, Clough R, Fan M, Hollis D, et al. Technical factors associated with radiation pneumonitis after local +/regional radiation therapy for breast cancer. Int J Radiat Oncol Biol Phys. 2002; 52: $137-43$.

19. Gokula K, Earnest A, Wong LC. Meta-analysis of incidence of early lung toxicity in 3-dimensional conformal irradiation of breast carcinomas. Radiat Oncol. 2013; 8: 268

20. Lingos TI, Recht A, Vicini F, Abner A, Silver B, Harris JR. Radiation pneumonitis in breast cancer patients treated with conservative surgery and radiation therapy. Int J Radiat Oncol Biol Phys. 1991; 21: 355-60.

21. Choi J, Kim YB, Shin KH, Ahn SJ, Lee HS, Park W, et al. Radiation Pneumonitis in Association with Internal Mammary Node Irradiation in Breast Cancer Patients: An Ancillary Result from the KROG 08-06 Study. J Breast Cancer. 2016; 19: 275-82.

22. Emami B, Lyman J, Brown A, Coia L, Goitein M, Munzenrider JE, et al. Tolerance of normal tissue to therapeutic irradiation. Int J Radiat Oncol Biol Phys. 1991; 21: 109-22.

23. Tsujino K, Hirota S, Endo M, Obayashi K, Kotani Y, Satouchi M, et al. Predictive value of dose-volume histogram parameters for predicting radiation pneumonitis after concurrent chemoradiation for lung cancer. Int J Radiat Oncol Biol Phys. 2003; 55: 110-5.

24. Koh ES, Sun A, Tran TH, Tsang R, Pintilie M, Hodgson DC, et al. Clinical dose-volume histogram analysis in predicting radiation pneumonitis in Hodgkin's lymphoma. Int J Radiat Oncol Biol Phys. 2006; 66: 223-8.

25. Graham MV, Purdy JA, Emami B, Harms W, Bosch W, Lockett MA, et al. Clinical dose-volume histogram analysis for pneumonitis after 3D treatment for non-small cell lung cancer (NSCLC). Int J Radiat Oncol Biol Phys. 1999; 45: 323-9.

26. Asakura H, Hashimoto T, Zenda S, Harada H, Hirakawa K, Mizumoto M, et al. Analysis of dose-volume histogram parameters for radiation pneumonitis after definitive concurrent chemoradiotherapy for esophageal cancer. Radiother Oncol. 2010; 95: 240-4.

27. Hernando ML, Marks LB, Bentel GC, Zhou SM, Hollis D, Das SK, et al Radiation-induced pulmonary toxicity: a dose-volume histogram analysis in 201 patients with lung cancer. Int J Radiat Oncol Biol Phys. 2001; 51: 650-9.

28. Fay M, Tan A, Fisher R, Mac Manus M, Wirth A, Ball D. Dose-volume histogram analysis as predictor of radiation pneumonitis in primary lung cancer patients treated with radiotherapy. Int J Radiat Oncol Biol Phys. 2005; 61: 1355-63.

29. Roeder F, Friedrich J, Timke C, Kappes J, Huber P, Krempien R, et al. Correlation of patient-related factors and dose-volume histogram parameters with the onset of radiation pneumonitis in patients with small cell lung cancer. Strahlenther Onkol. 2010; 186: 149-56.

30. Kutcher GJ, Burman C, Brewster L, Goitein M, Mohan R. Histogram reduction method for calculating complication probabilities for three-dimensional treatment planning evaluations. Int J Radiat Oncol Biol Phys. 1991; 21: 137-46. 
31. Blom-Goldman U, Svane G, Wennberg B, Lidestahl A, Lind PA. Quantitative assessment of lung density changes after 3-D radiotherapy for breast cancer. Acta Oncol. 2007; 46: 187-93.

32. Lind PA, Wennberg B, Gagliardi G, Fornander T. Pulmonary complications following different radiotherapy techniques for breast cancer, and the association to irradiated lung volume and dose. Breast Cancer Res Treat. 2001; 68: 199-210.

33. Yorke ED, Jackson A, Rosenzweig KE, Braban L, Leibel SA, Ling CC. Correlation of dosimetric factors and radiation pneumonitis for non-small-cell lung cancer patients in a recently completed dose escalation study. Int J Radiat Oncol Biol Phys. 2005; 63: 672-82

34. Wang S, Liao Z, Wei X, Liu HH, Tucker SL, Hu CS, et al. Analysis of clinical and dosimetric factors associated with treatment-related pneumonitis (TRP) in patients with non-small-cell lung cancer (NSCLC) treated with concurrent chemotherapy and three-dimensional conformal radiotherapy (3D-CRT). Int J Radiat Oncol Biol Phys. 2006; 66: 1399-407.

35. Schallenkamp JM, Miller RC, Brinkmann DH, Foote T, Garces YI. Incidence of radiation pneumonitis after thoracic irradiation: Dose-volume correlates. Int J Radiat Oncol Biol Phys. 2007; 67: 410-6. 